\title{
Analysis of the mass and width of the $X(4140)$ as axialvector tetraquark state
}

\author{
Zhi-Gang Wang a , Zun-Yan Di \\ Department of Physics, North China Electric Power University, Baoding 071003, People's Republic of China
}

Received: 30 November 2018 / Accepted: 11 January 2019 / Published online: 28 January 2019

(C) The Author(s) 2019

\begin{abstract}
In this article, we construct both the $[s c]_{T}[\bar{s} \bar{c}]_{A}+$ $[s c]_{A}[\bar{s} \bar{c}]_{T}$ type and $[s c]_{T}[\bar{s} \bar{c}]_{V}-[s c]_{V}[\bar{s} \bar{c}]_{T}$ type axialvector currents with $J^{P C}=1^{++}$to study the mass of the $X$ (4140) with the QCD sum rules. The predicted masses support assigning the $X(4140)$ to be the $[s c]_{T}[\bar{s} \bar{c}]_{V}-[s c]_{V}[\bar{s} \bar{c}]_{T}$ type axialvector tetraquark state. Then we study the hadronic coupling constant $g_{X J / \psi \phi}$ with the QCD sum rules based on solid quark-hadron duality, and obtain the decay width $\Gamma(X(4140) \rightarrow J / \psi \phi)=86.9 \pm 22.6 \mathrm{MeV}$, which is in excellent agreement with the experimental data $83 \pm$ $21_{-14}^{+21} \mathrm{MeV}$ from the $\mathrm{LHCb}$ collaboration.
\end{abstract}

\section{Introduction}

In 2009, the CDF collaboration observed the $X$ (4140) for the first time in the $J / \psi \phi$ mass spectrum in the exclusive $B^{+} \rightarrow J / \psi \phi K^{+}$decays in $p \bar{p}$ collisions with a statistical significance more than $3.8 \sigma$ [1]. Then the $X(4140)$ was confirmed by CDF, CMS, D0, LHCb collaborations [17]. The LHCb collaboration performed the first full amplitude analysis of the decays $B^{+} \rightarrow J / \psi \phi K^{+}$and confirmed the two old particles $X(4140)$ and $X(4274)$ in the $J / \psi \phi$ mass spectrum with statistical significances $8.4 \sigma$ and $6.0 \sigma$, respectively, and determined the spin-parity-changeconjugation to be $J^{P C}=1^{++}$with statistical significances $5.7 \sigma$ and $5.8 \sigma$, respectively [6,7]. In Table 1 , we present the mass, width, $J^{P C}$ of the $X$ (4140) from the different experiments. Although the width from the LHCb collaboration $[6,7]$ differs from other measurements greatly, the masses from different experiments are consistent with each other. The $D_{s}^{*} \bar{D}_{s}^{*}$ threshold is $4224.4 \mathrm{MeV}$ from the Particle Data Group [8], which leads to the possible molecule assignment for the $X(4140)$. The $X(4140)$ was observed in the final state $J / \psi \phi$, its $J^{P C}=0^{++}, 1^{++}, 2^{++}$for the S-wave couplings, and $0^{-+}, 1^{-+}, 2^{-+}, 3^{-+}$for the P-wave couplings. The

a e-mail: zgwang@aliyun.com most popular current to interpolate the $D_{s}^{*}$ meson is $J_{\alpha}(x)=$ $\bar{s}(x) \gamma_{\alpha} c(x)$, the most popular current to interpolate the $D_{s}^{*} \bar{D}_{s}^{*}$ molecular states is $J_{\alpha \beta}(x)=\bar{s}(x) \gamma_{\alpha} c(x) \bar{c}(x) \gamma_{\beta} s(x)$. We can study the $J^{P C}=0^{++}, 1^{-+}, 2^{++}, 1^{+-}, 1^{--} D_{s}^{*} \bar{D}_{s}^{*}$ molecular states with the QCD sum rules by using the suitable projectors. The LHCb collaboration determined the quantum numbers of the $X(4140)$ to be $J^{P C}=1^{++}$, which rules out the $0^{++}$or $2^{++} D_{s}^{*} \bar{D}_{s}^{*}$ molecule assignment, but does not rule out the existence of the $0^{++}$or $2^{++} D_{s}^{*} \bar{D}_{s}^{*}$ molecular states.

The possible assignments for the $X(4140)$ are tetraquark state [9-16], hybrid state [17-19] or rescattering effect [20], etc. In Ref. [9], Stancu calculates the mass spectrum of the $c \bar{c} s \bar{s}$ tetraquark states via a simple quark model with chromomagnetic interaction, and obtain two lowest masses $4195 \mathrm{MeV}$ and $4356 \mathrm{MeV}$ with $J^{P C}=1^{++}$. The value $4195 \mathrm{MeV}$ is consistent with the $\mathrm{LHCb}$ data $4146.5 \pm$ $4.5_{-2.8}^{+4.6} \mathrm{MeV}[6,7]$. In the simple chromomagnetic interaction model, there are no correlated quarks or diquarks [9]. In Ref. [11], Lebed and Polosa assign the $X(4140)$ to be the $J^{P C}=1^{++}$diquark-antidiquark state $[c s]_{A}[\bar{c} \bar{s}]_{S}+$ $[c s]_{S}[\bar{c} \bar{s}]_{A}$ based on the effective Hamiltonian with the spinspin and spin-orbit interactions, then in Ref. [12], L. Maiani, A. D. Polosa and V. Riquer take the mass of the $X(4140)$ as input parameter, and obtain the mass spectrum of the $c \bar{c} s \bar{s}$ tetraquark states with positive parity, however, they observe that there is no room for the $X$ (4274), and suggest the $X$ (4274) corresponds to two, almost degenerate, unresolved lines with $J^{P C}=0^{++}$and $2^{++}$.

In the QCD sum rules, we usually take the diquarks (or correlations) and antidiquarks (or correlations) as the basic constituents to construct the interpolating currents, the predictions can be compared to that based on the diquarkantidiquark model directly $[11,12]$. In the quantum field theory, the diquark operators (or diquarks) $\varepsilon^{i j k} q_{j}^{T} C \Gamma q_{k}^{\prime}$ have five structures in Dirac spinor space, where the $i, j$ and $k$ are color indexes, $C \Gamma=C \gamma_{5}, C, C \gamma_{\mu} \gamma_{5}, C \gamma_{\mu}$ and $C \sigma_{\mu \nu}$ for the 
Table 1 The mass, width, $J^{P C}$ of the $X(4140)$ from the different experiments
Table 2 The masses of the $s c \bar{s} \bar{c}$ tetraquark states relevant to the $X$ (4140) from the QCD sum rules, the OPE denotes truncations of the operator product expansion up to the vacuum condensates of dimension $n$, the No denotes the vacuum condensates of dimension $n^{\prime}$ are not included

\begin{tabular}{|c|c|c|c|c|c|}
\hline Year & Mass $(\mathrm{MeV})$ & Width $(\mathrm{MeV})$ & $J^{P C}$ & Significance & Experiment \\
\hline 2009 & $4143.0 \pm 2.9 \pm 1.2$ & \multicolumn{2}{|c|}{$11.7_{-5.0}^{+8.3} \pm 3.7$} & $3.8 \sigma$ & $\mathrm{CDF}[1]$ \\
\hline 2011 & $4143.4_{-3.0}^{+2.9} \pm 0.6$ & \multicolumn{2}{|c|}{$15.3_{-6.1}^{+10.4} \pm 2.5$} & $5.0 \sigma$ & $\mathrm{CDF}[2]$ \\
\hline 2013 & $4148.0 \pm 2.4 \pm 6.3$ & \multicolumn{2}{|c|}{$28_{-11}^{+15} \pm 19$} & $5.0 \sigma$ & CMS [3] \\
\hline 2013 & $4159.0 \pm 4.3 \pm 6.6$ & \multicolumn{2}{|c|}{$19.9 \pm 12.6_{-8.0}^{+3.0}$} & $3.1 \sigma$ & D0 [4] \\
\hline 2015 & $4152.5 \pm 1.7_{-5.4}^{+6.2}$ & \multicolumn{2}{|c|}{$16.3 \pm 5.6 \pm 11.4$} & $4.7 \sigma$ & D0 [5] \\
\hline 2016 & $4146.5 \pm 4.5_{-2.8}^{+4.6}$ & \multicolumn{2}{|c|}{$83 \pm 21_{-14}^{+21}$} & $8.4 \sigma$ & $\mathrm{LHCb}[6,7]$ \\
\hline$\overline{J^{P C}}$ & Structures & OPE (No) & $\operatorname{mass}(\mathrm{GeV})$ & Assignment & $\overline{\text { References }}$ \\
\hline $0^{++}$ & {$[s c]_{A}[\bar{s} \bar{c}]_{A}$} & 10 & $3.92 / 4.50$ & $X(3915) / X(4500)$ & [21] \\
\hline $0^{++}$ & {$[s c]_{V}[\bar{s} \bar{c}]_{V}$} & 10 & 4.70 & $X(4700)$ & [21] \\
\hline $0^{++}$ & {$[s c]_{A}[\bar{s} \bar{c}]_{A}$} & 10 & 3.98 & $?$ & [10] \\
\hline $0^{++}$ & {$[s c]_{S}[\bar{s} \bar{c}]_{S}$} & 10 & $3.89 / 4.35$ & $X(3915) / ?$ & [22] \\
\hline $0^{++}$ & {$[s c]_{P}[\bar{s} \bar{c}]_{P}$} & 10 & 5.48 & $?$ & [22] \\
\hline $1^{++}$ & {$[s c]_{S}[\bar{s} \bar{c}]_{A}+[s c]_{A}[\bar{s} \bar{c}]_{S}$} & 10 & 3.95 & $?$ & [23] \\
\hline $1^{++}$ & {$[s c]_{P}[\bar{s} \bar{c}]_{V}+[s c]_{V}[\bar{s} \bar{c}]_{P}$} & 10 & 5.00 & $?$ & [23] \\
\hline $1^{++}$ & {$[s c]_{S}[\bar{s} \bar{c}]_{A}+[s c]_{A}[\bar{s} \bar{c}]_{S}$} & $8(7)$ & 4.07 & $X(4140)$ & [24] \\
\hline $1^{++}$ & {$[s c]_{S}[\bar{s} \bar{c}]_{A}+[s c]_{A}[\bar{s} \bar{c}]_{S}$} & 8 & 4.18 & $X(4140)$ & [26] \\
\hline $2^{++}$ & {$[s c]_{A}[\bar{s} \bar{c}]_{A}$} & 10 & 4.13 & $? X(4140)$ & {$[10]$} \\
\hline
\end{tabular}

scalar $(S)$, pseudoscalar $(P)$, vector $(V)$, axialvector $(A)$ and tensor ( $T$ ) diquarks, respectively. The $C \gamma_{5}$ and $C \gamma_{\mu}$ diquark states have the spin-parity $J^{P}=0^{+}$and $1^{+}$, respectively, the $C$ and $C \gamma_{\mu} \gamma_{5}$ diquark states have the spin-parity $J^{P}=0^{-}$ and $1^{-}$, respectively, the $C \sigma_{\mu \nu}$ and $C \sigma_{\mu \nu} \gamma_{5}$ diquark states (or operators) have both the $J^{P}=1^{+}$and $1^{-}$components. The relevant diquark-antidiquark type scalar, axialvector and tensor tetraquark states $s c \bar{s} \bar{c}$ have been studied with the QCD sum rules [10,21-27], see Table 2.

In the QCD sum rules for the hidden-charm (or hiddenbottom) tetraquark states and molecular states, the integrals

$\int_{4 m_{Q}^{2}(\mu)}^{s_{0}} d s \rho_{Q C D}(s, \mu) \exp \left(-\frac{s}{T^{2}}\right)$

are sensitive to the energy scales $\mu$, where the $\rho_{Q C D}(s, \mu)$ are the QCD spectral densities, the $T^{2}$ are the Borel parameters, the $s_{0}$ are the continuum threshold parameters, the predicted masses depend heavily on the energy scales $\mu$. In Refs. [28-31], we suggest an energy scale formula $\mu=$ $\sqrt{M_{X / Y / Z}^{2}-\left(2 \mathbb{M}_{Q}\right)^{2}}$ with the effective $Q$-quark mass $\mathbb{M}_{Q}$ to determine the ideal energy scales of the QCD spectral densities. The formula enhances the pole contributions remarkably, we obtain the pole contributions as large as $(40-60) \%$ in Refs. [10,21-23], otherwise, the pole contributions are about $40 \%$ [24] or $20 \%$ [26,27]. The energy scale formula also works well in the QCD sum rules for the hidden-charm pentaquark states [32-34].
From Table 2, we can see that the $[s c]_{S}[\bar{s} \bar{c}]_{A}+[s c]_{A}[\bar{s} \bar{c}]_{S}$ type axialvector current cannot reproduce the mass of the $X(4140)$ if the pole dominance criterion is satisfied. If we take energy scale formula $\mu=\sqrt{M_{X / Y / Z}^{2}-\left(2 \mathbb{M}_{c}\right)^{2}}$ and choose the updated value $\mathbb{M}_{c}=1.82 \mathrm{GeV}$ [35], we can obtain the optimal energy scales $\mu=1.4 \mathrm{GeV}$ and $2.0 \mathrm{GeV}$ for the QCD spectral densities in the QCD sum rules for the $Z_{c}(3900)$ and $X(4140)$, respectively. In Ref. [23], we observe that the mass of the $X(4140)$ can be reproduced at the energy scale $\mu=1.1 \mathrm{GeV}$, a too low energy scale. The QCD sum rules do not support assigning the $X(4140)$ to be the $[s c]_{S}[\bar{s} \bar{c}]_{A}+[s c]_{A}[\bar{s} \bar{c}]_{S}$ type axialvector tetraquark state.

In this article, we construct the $[s c]_{T}[\bar{s} \bar{c}]_{A}+[s c]_{A}[\bar{s} \bar{c}]_{T}$ type and $[s c]_{T}[\bar{s} \bar{c}]_{V}-[s c]_{V}[\bar{s} \bar{c}]_{T}$ type axialvector currents to study the mass of the $X(4140)$ as the axialvector tetraquark state with the QCD sum rules in details, then study the width of the $X(4140)$ with the QCD sum rules based on the solid quark-hadron duality.

The article is arranged as follows: we derive the QCD sum rules for the mass and width of the $X(4140)$ as axialvector tetraquark state in Sects. 2 and 3 respectively; Sect. 4 is reserved for our conclusion.

\section{The mass of the $X(4140)$ as the axialvector tetraquark state}

In the following, we write down the two-point correlation functions $\Pi_{\mu \mu^{\prime}}(p)$ in the QCD sum rules, 
$\Pi_{\mu \mu^{\prime}}(p)=i \int d^{4} x e^{i p \cdot x}\left\langle 0\left|T\left\{J_{\mu}(x) J_{\mu^{\prime}}^{\dagger}(0)\right\}\right| 0\right\rangle$,

where $J_{\mu}(x)=J_{\mu}^{1}(x), J_{\mu}^{2}(x)$,

$$
\begin{aligned}
J_{\mu}^{1}(x)= & \frac{\varepsilon^{i j k} \varepsilon^{i m n}}{\sqrt{2}}\left[s^{T j}(x) C \sigma_{\mu \nu} \gamma_{5} c^{k}(x) \bar{s}^{m}(x) \gamma^{\nu} C \bar{c}^{T n}(x)\right. \\
& \left.+s^{T j}(x) C \gamma^{v} c^{k}(x) \bar{s}^{m}(x) \gamma_{5} \sigma_{\mu \nu} C \bar{c}^{T n}(x)\right], \\
J_{\mu}^{2}(x)= & \frac{\varepsilon^{i j k} \varepsilon^{i m n}}{\sqrt{2}}\left[s^{T j}(x) C \sigma_{\mu \nu} c^{k}(x) \bar{s}^{m}(x) \gamma_{5} \gamma^{\nu} C \bar{c}^{T n}(x)\right. \\
& \left.-s^{T j}(x) C \gamma^{v} \gamma_{5} c^{k}(x) \bar{s}^{m}(x) \sigma_{\mu \nu} C \bar{c}^{T n}(x)\right],
\end{aligned}
$$

the $i, j, k, m, n$ are color indexes. Under charge conjugation (parity) transform $\widehat{C}(\widehat{P})$, the currents $J_{\mu}(x)$ have the properties,

$$
\begin{aligned}
& \widehat{C} J_{\mu}(x) \widehat{C}^{-1}=+J_{\mu}(x), \\
& \widehat{P} J_{\mu}(x) \widehat{P}^{-1}=-J^{\mu}(\tilde{x}),
\end{aligned}
$$

the four vectors $x^{\mu}=(t, \mathbf{x})$ and $\tilde{x}^{\mu}=(t,-\mathbf{x})$. The currents $J_{\mu}^{1}(x)$ and $J_{\mu}^{2}(x)$ couple potentially to the $[s c]_{T}[\bar{s} \bar{c}]_{A}+$ $[s c]_{V}[\bar{s} \bar{c}]_{A}$ and $[s c]_{T}[\bar{s} \bar{c}]_{V}-[s c]_{V}[\bar{s} \bar{c}]_{T}$ axialvector tetraquark states with $J^{P C}=1^{++}$, respectively. The tensor diquark operators have the properties,

$$
\begin{aligned}
\widehat{P} \varepsilon^{i j k} s^{T j}(x) C \sigma_{\mu \nu} \gamma_{5} c^{k}(x) \widehat{P}^{-1} & =\varepsilon^{i j k} s^{T j}(\tilde{x}) C \sigma^{\mu \nu} \gamma_{5} c^{k}(\tilde{x}), \\
\widehat{P} \varepsilon^{i j k}{ }^{T j}(x) C \sigma_{\mu \nu} c^{k}(x) \widehat{P}^{-1} & =-\varepsilon^{i j k} s^{T j}(\tilde{x}) C \sigma^{\mu \nu} c^{k}(\tilde{x}),
\end{aligned}
$$

under parity transform, the tensor diquark operators couple potentially to both the $J^{P}=1^{+}$and $1^{-}$diquark states. We should project out the $1^{+}$or $1^{-}$component by multiplying tensor diquark operators by the axialvector antidiquark operator $\varepsilon^{i m n} \bar{s}^{m}(x) \gamma^{\nu} C \bar{c}^{T n}(x)$ or vector antidiquark operator $\varepsilon^{i m n} \bar{s}^{m}(x) \gamma_{5} \gamma^{\nu} C \bar{c}^{T n}(x)$.

At the phenomenological side, we insert a complete set of intermediate hadronic states with the same quantum numbers as the current operators $J_{\mu}(x)$ into the correlation functions $\Pi_{\mu \mu^{\prime}}(p)$ to obtain the hadronic representation [36-38], and isolate the ground state contributions,

$$
\begin{aligned}
\Pi_{\mu \mu^{\prime}}(p) & =\frac{\lambda_{X}^{2}}{M_{X}^{2}-p^{2}}\left(-g_{\mu \mu^{\prime}}+\frac{p_{\mu} p_{\mu^{\prime}}}{p^{2}}\right)+\cdots \\
& =\Pi\left(p^{2}\right)\left(-g_{\mu \mu^{\prime}}+\frac{p_{\mu} p_{\mu^{\prime}}}{p^{2}}\right)+\cdots,
\end{aligned}
$$

where the pole residues $\lambda_{X}$ are defined by $\left\langle 0\left|J_{\mu}(0)\right| X(p)\right\rangle=$ $\lambda_{X} \varepsilon_{\mu}$, the $\varepsilon_{\mu}$ are the polarization vectors of the axialvector tetraquark states $X$.

Now we briefly outline the operator product expansion for the correlation functions $\Pi_{\mu \mu^{\prime}}(p)$. We contract the quark fields $s$ and $c$ in the correlation functions $\Pi_{\mu \mu^{\prime}}(p)$ with Wick theorem, and obtain the results,

$$
\begin{aligned}
& \Pi_{\mu \mu^{\prime}}^{1}(p)=-\frac{i}{2} \varepsilon^{i j k} \varepsilon^{i m n} \varepsilon^{i^{\prime} j^{\prime} k^{\prime}} \varepsilon^{i^{\prime} m^{\prime} n^{\prime}} \int d^{4} x e^{i p \cdot x} \\
& \left\{\operatorname{Tr}\left[\sigma_{\mu \nu} \gamma_{5} S_{c}^{k k^{\prime}}(x) \gamma_{5} \sigma_{\mu^{\prime} \nu^{\prime}} C S^{T j j^{\prime}}(x) C\right]\right. \\
& \times \operatorname{Tr}\left[\gamma^{v^{\prime}} S_{c}^{n^{\prime} n}(-x) \gamma^{\nu} C S^{T m^{\prime} m}(-x) C\right] \\
& +\operatorname{Tr}\left[\gamma^{v} S_{c}^{k k^{\prime}}(x) \gamma_{5} \sigma_{\mu^{\prime} \nu^{\prime}} C S^{T j j^{\prime}}(x) C\right] \\
& \times \operatorname{Tr}\left[\gamma^{\nu^{\prime}} S_{c}^{n^{\prime} n}(-x) \gamma_{5} \sigma_{\mu \nu} C S^{T m^{\prime} m}(-x) C\right] \\
& +\operatorname{Tr}\left[\sigma_{\mu \nu} \gamma_{5} S_{c}^{k k^{\prime}}(x) \gamma^{\nu^{\prime}} C S^{T j j^{\prime}}(x) C\right] \\
& \times \operatorname{Tr}\left[\sigma_{\mu^{\prime} \nu^{\prime} \gamma_{5}} S_{c}^{n^{\prime} n}(-x) \gamma^{\nu} C S^{T m^{\prime} m}(-x) C\right] \\
& +\operatorname{Tr}\left[\gamma^{\nu} S_{c}^{k k^{\prime}}(x) \gamma^{v^{\prime}} C S^{T j j^{\prime}}(x) C\right] \\
& \left.\times \operatorname{Tr}\left[\sigma_{\mu^{\prime} \nu^{\prime}} \gamma_{5} S_{c}^{n^{\prime} n}(-x) \gamma_{5} \sigma_{\mu \nu} C S^{T m^{\prime} m}(-x) C\right]\right\}, \\
& \Pi_{\mu \mu^{\prime}}^{2}(p)=-\frac{i}{2} \varepsilon^{i j k} \varepsilon^{i m n} \varepsilon^{i^{\prime} j^{\prime} k^{\prime}} \varepsilon^{i^{\prime} m^{\prime} n^{\prime}} \int d^{4} x e^{i p \cdot x} \\
& \left\{\operatorname{Tr}\left[\sigma_{\mu \nu} S_{c}^{k k^{\prime}}(x) \sigma_{\mu^{\prime} \nu^{\prime}} C S^{T j j^{\prime}}(x) C\right]\right. \\
& \times \operatorname{Tr}\left[\gamma^{v^{\prime}} \gamma_{5} S_{c}^{n^{\prime} n}(-x) \gamma_{5} \gamma^{\nu} C S^{T m^{\prime} m}(-x) C\right] \\
& +\operatorname{Tr}\left[\gamma^{v} \gamma_{5} S_{c}^{k k^{\prime}}(x) \sigma_{\mu^{\prime} \nu^{\prime}} C S^{T j j^{\prime}}(x) C\right] \\
& \times \operatorname{Tr}\left[\gamma^{\nu^{\prime}} \gamma_{5} S_{c}^{n^{\prime} n}(-x) \sigma_{\mu \nu} C S^{T m^{\prime} m}(-x) C\right] \\
& +\operatorname{Tr}\left[\sigma_{\mu \nu} S_{c}^{k k^{\prime}}(x) \gamma_{5} \gamma^{\nu^{\prime}} C S^{T j j^{\prime}}(x) C\right] \\
& \times \operatorname{Tr}\left[\sigma_{\mu^{\prime} \nu^{\prime}} S_{c}^{n^{\prime} n}(-x) \gamma_{5} \gamma^{\nu} C S^{T m^{\prime} m}(-x) C\right] \\
& +\operatorname{Tr}\left[\gamma^{\nu} \gamma_{5} S_{c}^{k k^{\prime}}(x) \gamma_{5} \gamma^{\nu^{\prime}} C S^{T j j^{\prime}}(x) C\right] \operatorname{Tr} \\
& \left.\times\left[\sigma_{\mu^{\prime} \nu^{\prime}} S_{c}^{n^{\prime} n}(-x) \sigma_{\mu \nu} C S^{T m^{\prime} m}(-x) C\right]\right\},
\end{aligned}
$$

where

$$
\begin{aligned}
S^{i j}(x)= & \frac{i \delta_{i j} \not x}{2 \pi^{2} x^{4}}-\frac{\delta_{i j} m_{s}}{4 \pi^{2} x^{2}}-\frac{\delta_{i j}\langle\bar{s} s\rangle}{12}+\frac{i \delta_{i j} \not m_{s}\langle\bar{s} s\rangle}{48} \\
& -\frac{\delta_{i j} x^{2}\left\langle\bar{s} g_{s} \sigma G s\right\rangle}{192}+\frac{i \delta_{i j} x^{2} \not \not m_{s}\left\langle\bar{s} g_{s} \sigma G s\right\rangle}{1152} \\
& -\frac{i g_{s} G_{\alpha \beta}^{a} t_{i j}^{a}\left(\not \not \sigma^{\alpha \beta}+\sigma^{\alpha \beta} \not x\right)}{32 \pi^{2} x^{2}}-\frac{\delta_{i j} x^{4}\langle\bar{s} s\rangle\left\langle g_{s}^{2} G G\right\rangle}{27648} \\
& -\frac{1}{8}\left\langle\bar{s}_{j} \sigma^{\mu v} s_{i}\right\rangle \sigma_{\mu \nu}+\cdots, \\
S_{c}^{i j}(x)= & \frac{i}{(2 \pi)^{4}} \int d^{4} k e^{-i k \cdot x}\left\{\frac{\delta_{i j}}{\not k-m_{c}}\right. \\
& -\frac{g_{s} G_{\alpha \beta}^{n} t_{i j}^{n} \frac{\sigma^{\alpha \beta}\left(\not k+m_{c}\right)+\left(\not k+m_{c}\right) \sigma^{\alpha \beta}}{4}}{\left(k^{2}-m_{c}^{2}\right)^{2}} \\
& \left.-\frac{g_{s}^{2}\left(t^{a} t^{b}\right)_{i j} G_{\alpha \beta}^{a} G_{\mu \nu}^{b}\left(f^{\alpha \beta \mu \nu}+f^{\alpha \mu \beta \nu}+f^{\alpha \mu \nu \beta}\right)}{4\left(k^{2}-m_{c}^{2}\right)^{5}}+\cdots\right\},
\end{aligned}
$$


$f^{\lambda \alpha \beta}=\left(\not k+m_{c}\right) \gamma^{\lambda}\left(\not k+m_{c}\right) \gamma^{\alpha}\left(\not k+m_{c}\right) \gamma^{\beta}\left(\not k+m_{c}\right)$, $f^{\alpha \beta \mu \nu}=\left(\not k+m_{c}\right) \gamma^{\alpha}\left(\not k+m_{c}\right) \gamma^{\beta}\left(\not k+m_{c}\right) \gamma^{\mu}\left(\not k+m_{c}\right) \gamma^{\nu}\left(\not k+m_{c}\right)$,

and $t^{n}=\frac{\lambda^{n}}{2}$ [38], then compute the integrals both in the coordinate space and in the momentum space, and obtain the correlation functions $\Pi_{\mu \mu^{\prime}}(p)$ (i.e. $\Pi_{\mu \mu^{\prime}}^{1}(p)$ and $\left.\Pi_{\mu \mu^{\prime}}^{2}(p)\right)$, therefore the QCD spectral densities through dispersion relation $\rho(s)=\lim _{\varepsilon \rightarrow 0} \frac{\operatorname{Im} \Pi(s+i \varepsilon)}{\pi}$. For technical details, one can consult Ref. [39].

Now we take the quark-hadron duality below the continuum thresholds $s_{0}$ and perform Borel transform with respect to the variable $P^{2}=-p^{2}$ to obtain the QCD sum rules:

$\lambda_{X}^{2} \exp \left(-\frac{M_{X}^{2}}{T^{2}}\right)=\int_{4 m_{c}^{2}}^{s_{0}} d s \rho(s) \exp \left(-\frac{s}{T^{2}}\right)$,

where $\rho(s)=\rho_{T A}(s)$ and $\rho_{T V}(s)$ for the $[s c]_{T}[\bar{s} \bar{c}]_{A}+$ $[s c]_{V}[\bar{s} \bar{c}]_{A}$ and $[s c]_{T}[\bar{s} \bar{c}]_{V}-[s c]_{V}[\bar{s} \bar{c}]_{T}$ axialvector tetraquark states respectively,

$$
\begin{aligned}
\rho_{T A}(s)= & \rho_{0}(s)+\rho_{3}(s)+\rho_{4}(s)+\rho_{5}(s)+\rho_{6}(s)+\rho_{7}(s) \\
& +\rho_{8}(s)+\rho_{10}(s), \\
\rho_{T V}(s)= & \left.\rho_{T A}(s)\right|_{m_{c} \rightarrow-m_{c}},
\end{aligned}
$$

the subscripts $i$ in the QCD spectral densities $\rho_{i}(s)$ denote the dimensions of the vacuum condensates,

$$
\begin{aligned}
\rho_{3}(s) & \propto\langle\bar{s} s\rangle \\
\rho_{4}(s) & \propto\left\langle\frac{\alpha_{s} G G}{\pi}\right\rangle \\
\rho_{5}(s) & \propto\left\langle\bar{s} g_{s} \sigma G s\right\rangle \\
\rho_{6}(s) & \propto\langle\bar{s} s\rangle^{2} \\
\rho_{7}(s) & \propto\langle\bar{s} s\rangle\left\langle\frac{\alpha_{s} G G}{\pi}\right\rangle \\
\rho_{8}(s) & \propto\langle\bar{s} s\rangle\left\langle\bar{s} g_{s} \sigma G s\right\rangle \\
\rho_{10}(s) & \propto\left\langle\bar{s} g_{s} \sigma G s\right\rangle^{2},\langle\bar{s} s\rangle^{2}\left\langle\frac{\alpha_{s} G G}{\pi}\right\rangle,
\end{aligned}
$$

the lengthy expressions of the QCD spectral densities are given in Appendix.

We derive Eq. (11) with respect to $\tau=\frac{1}{T^{2}}$, then eliminate the pole residues $\lambda_{X}$ to obtain the QCD sum rules for the masses,

$$
M_{X}^{2}=-\frac{\int_{4 m_{c}^{2}}^{s_{0}} d s \frac{d}{d \tau} \rho(s) e^{-\tau s}}{\int_{4 m_{c}^{2}}^{s_{0}} d s \rho(s) e^{-\tau s}} .
$$

At the QCD side, we take the vacuum condensates to be the standard values $\langle\bar{q} q\rangle=-(0.24 \pm 0.01 \mathrm{GeV})^{3},\langle\bar{s} s\rangle=(0.8 \pm$ $0.1)\langle\bar{q} q\rangle,\left\langle\bar{s} g_{s} \sigma G s\right\rangle=m_{0}^{2}\langle\bar{s} s\rangle, m_{0}^{2}=(0.8 \pm 0.1) \mathrm{GeV}^{2}$, $\left\langle\frac{\alpha_{s} G G}{\pi}\right\rangle=(0.33 \mathrm{GeV})^{4}$ at the energy scale $\mu=1 \mathrm{GeV}$ [36-38,40], and take the $\overline{M S}$ masses $m_{c}\left(m_{c}\right)=(1.275 \pm$ $0.025) \mathrm{GeV}$ and $m_{s}(\mu=2 \mathrm{GeV})=(0.095 \pm 0.005) \mathrm{GeV}$ from the Particle Data Group [8]. Moreover, we take into account the energy-scale dependence of the quark condensate, mixed quark condensate and $\overline{M S}$ masses from the renormalization group equation,

$$
\langle\bar{s} s\rangle(\mu)=\langle\bar{s} s\rangle(Q)\left[\frac{\alpha_{s}(Q)}{\alpha_{s}(\mu)}\right]^{\frac{12}{33-2 n_{f}}},
$$

$$
\begin{aligned}
\left\langle\bar{s} g_{s} \sigma G s\right\rangle(\mu) & =\left\langle\bar{s} g_{s} \sigma G s\right\rangle(Q)\left[\frac{\alpha_{s}(Q)}{\alpha_{s}(\mu)}\right]^{\frac{2}{33-2 n_{f}}}, \\
m_{c}(\mu)= & m_{c}\left(m_{c}\right)\left[\frac{\alpha_{s}(\mu)}{\alpha_{s}\left(m_{c}\right)}\right]^{\frac{12}{33-2 n_{f}}}, \\
m_{s}(\mu)= & m_{s}(2 \mathrm{GeV})\left[\frac{\alpha_{s}(\mu)}{\alpha_{s}(2 \mathrm{GeV})}\right]^{\frac{12}{33-2 n_{f}}}, \\
\alpha_{s}(\mu)= & \frac{1}{b_{0} t}\left[1-\frac{b_{1}}{b_{0}^{2}} \frac{\log t}{t}\right. \\
& \left.+\frac{b_{1}^{2}\left(\log ^{2} t-\log t-1\right)+b_{0} b_{2}}{b_{0}^{4} t^{2}}\right],
\end{aligned}
$$

where $t=\log \frac{\mu^{2}}{\Lambda^{2}}, b_{0}=\frac{33-2 n_{f}}{12 \pi}, b_{1}=\frac{153-19 n_{f}}{24 \pi^{2}}$, $b_{2}=\frac{2857-\frac{5033}{9} n_{f}+\frac{325}{27} n_{f}^{2}}{128 \pi^{3}}, \Lambda=210 \mathrm{MeV}, 292 \mathrm{MeV}$ and $332 \mathrm{MeV}$ for the flavors $n_{f}=5,4$ and 3 , respectively $[8,41]$, and evolve all the input parameters to the typical energy scales $\mu$ satisfying the energy scale formula $\mu=$ $\sqrt{M_{X / Y / Z}^{2}-\left(2 \mathbb{M}_{c}\right)^{2}}$ to extract the masses of the axialvector tetraquark states.

We search for the optimal Borel parameters $T^{2}$ and threshold parameters $s_{0}$ to satisfy the following four criteria:

1. Pole dominance at the phenomenological side;

2. Convergence of the operator product expansion;

3. Appearance of the Borel platforms;

4. Satisfying the energy scale formula,

via try and error, and obtain the Borel windows $T^{2}$, threshold parameters $s_{0}$, optimal energy scales of the QCD spectral densities, and pole contributions of the ground states, see Table 3.

Now we take a short digression to illustrate how to impose the four criteria to choose the Borel parameters $T^{2}$ and continuum threshold parameters $s_{0}$. Firstly, we set $M_{X}=3.9 \mathrm{GeV}$ tentatively, and obtain the energy scale $\mu=1.4 \mathrm{GeV}$ according to the energy scale formula. Then we take the continuum threshold parameters to be $\sqrt{s_{0}}=$ $(3.9+0.5) \mathrm{GeV}$ as the energy gap between the ground state and the first radial excited state is about $(0.4-0.6) \mathrm{GeV}$, and obtain the predicted masses $M_{X}$, pole contributions, and the contributions of the vacuum condensates of dimension 10 . We observe that the predicted masses $M_{X}$ are much larger than $3.9 \mathrm{GeV}$ and the pole contributions are much smaller than $50 \%$ in the regions where the Borel platforms appear, 
Table 3 The Borel windows, continuum threshold parameters, ideal energy scales, pole contributions, masses and pole residues for the axialvector tetraquark states

\begin{tabular}{lllllll}
\hline & $T^{2}\left(\mathrm{GeV}^{2}\right)$ & $\sqrt{s_{0}}(\mathrm{GeV})$ & $\mu(\mathrm{GeV})$ & Pole & $M(\mathrm{GeV})$ & $\lambda\left(\mathrm{GeV}^{5}\right)$ \\
\hline$J_{\mu}^{1}(x)$ & $4.4-5.0$ & $5.7 \pm 0.1$ & 3.7 & $(40-60) \%$ & $5.20 \pm 0.11$ & $(2.01 \pm 0.24) \times 10^{-1}$ \\
$J_{\mu}^{2}(x)$ & $2.7-3.3$ & $4.7 \pm 0.1$ & 2.0 & $(41-69) \%$ & $4.14 \pm 0.10$ & $(4.30 \pm 0.85) \times 10^{-2}$
\end{tabular}

furthermore, the contributions of the vacuum condensates of dimension 10 are not small enough. Then we choose the masses $M_{X}>3.9 \mathrm{GeV}$, say $M_{X}=4.0 \mathrm{GeV}, 4.1 \mathrm{GeV}, \ldots$ and reiterate the same procedure until obtain the optimal Borel parameters $T^{2}$ and continuum threshold parameters $s_{0}$ satisfying the four criteria.

From Table 3, we can see that the pole dominance criterion is well satisfied. In calculations, we observe that the contributions of the vacuum condensates of dimension 10 are $\ll 1 \%$ (about $1 \%$ ) in the QCD sum rules for the current $J_{\mu}^{1}(x)\left(J_{\mu}^{2}(x)\right)$, the operator product expansion is well convergent. We take into account all uncertainties of the input parameters, and obtain the values of the masses and pole residues of the axialvector tetraquark states, see Table 3 and Figs. 1, 2. From Table 3, we can see that the energy scale formula is well satisfied. From Figs. 1, 2 and Table 3, we can see that there appear platforms in the Borel windows. The four criteria are all satisfied, our predictions are reliable.

From Table 3, we can see the predicted mass $M_{X}=$ $4.14 \pm 0.10 \mathrm{GeV}$ for the $[s c]_{T}[\bar{s} \bar{c}]_{V}-[s c]_{V}[\bar{s} \bar{c}]_{T}$ axialvector tetraquark state is in excellent agreement with the experimental data $4146.5 \pm 4.5_{-2.8}^{+4.6} \mathrm{MeV}$ from the $\mathrm{LHCb}$ collaboration $[6,7]$, which supports assigning the $X(4140)$ to be the $[s c]_{T}[\bar{s} \bar{c}]_{V}-[s c]_{V}[\bar{s} \bar{c}]_{T}$ tetraquark state with $J^{P C}=1^{++}$. While the $[s c]_{T}[\bar{s} \bar{c}]_{A}+[s c]_{V}[\bar{s} \bar{c}]_{A}$ axialvector tetraquark state has a much larger mass than that of the $X(4140)$.

\section{The width of the $X(4140)$ as the axialvector tetraquark state}

We can study the two-body strong decay $X(4140) \rightarrow J / \psi \phi$ with the three-point correlation function $\Pi_{\alpha \beta \mu}(p, q)$,

$$
\begin{aligned}
& \Pi_{\alpha \beta \mu}(p, q) \\
& \quad=i^{2} \int d^{4} x d^{4} y e^{i p x} e^{i q y}\left\langle 0\left|T\left\{J_{\alpha}^{J / \psi}(x) J_{\beta}^{\phi}(y) J_{\mu}^{\dagger}(0)\right\}\right| 0\right\rangle,
\end{aligned}
$$

where the currents

$$
\begin{aligned}
J_{\alpha}^{J / \psi}(x) & =\bar{c}(x) \gamma_{\alpha} c(x), \\
J_{\beta}^{\phi}(y) & =\bar{s}(y) \gamma_{\beta} s(y), \\
J_{\mu}(0) & =J_{\mu}^{2}(0),
\end{aligned}
$$

interpolate the mesons $J / \psi, \phi(1020)$ and $X(4140)$ respectively,

$$
\begin{aligned}
\left\langle 0\left|J_{\alpha}^{J / \psi}(0)\right| J / \psi(p)\right\rangle & =f_{J / \psi} m_{J / \psi} \xi_{\alpha}, \\
\left\langle 0\left|J_{\beta}^{\phi}(0)\right| \phi(q)\right\rangle & =f_{\phi} m_{\phi} \zeta_{\beta},
\end{aligned}
$$

the $f_{J / \psi}$ and $f_{\phi}$ are the decay constants, the $\xi_{\alpha}$ and $\zeta_{\beta}$ are polarization vectors of the mesons $J / \psi$ and $\phi(1020)$, respectively. In this section, we will use the notation $m_{X}$ in stead of $M_{X}$ for the special case $J_{\mu}(0)=J_{\mu}^{2}(0)$.

At the phenomenological side, we insert a complete set of intermediate hadronic states with the same quantum numbers as the current operators $J_{\alpha}^{J / \psi}(x), J_{\beta}^{\phi}(y), J_{\mu}^{\dagger}(0)$ into the threepoint correlation function $\Pi_{\alpha \beta \mu}(p, q)$ and isolate the ground state contributions to obtain the result,

$$
\begin{aligned}
& \Pi_{\alpha \beta \mu}(p, q) \\
& =\frac{f_{\phi} m_{\phi} f_{J / \psi} m_{J / \psi} \lambda_{X} g_{X J / \psi \phi}}{\left(m_{X}^{2}-p^{\prime 2}\right)\left(m_{J / \psi}^{2}-p^{2}\right)\left(m_{\phi}^{2}-q^{2}\right)} \varepsilon^{\lambda \tau \rho \theta} p_{\lambda}^{\prime}\left(-g_{\mu \tau}\right. \\
& \left.+\frac{p_{\mu}^{\prime} p_{\tau}^{\prime}}{p^{\prime 2}}\right)\left(-g_{\alpha \rho}+\frac{p_{\alpha} p_{\rho}}{p^{2}}\right)\left(-g_{\beta \theta}+\frac{q_{\beta} q_{\theta}}{q^{2}}\right)+\cdots \\
& =\left\{\frac{f_{\phi} m_{\phi} f_{J / \psi} m_{J / \psi} \lambda_{X} g_{X J / \psi \phi}}{\left(m_{X}^{2}-p^{\prime 2}\right)\left(m_{J / \psi}^{2}-p^{2}\right)\left(m_{\phi}^{2}-q^{2}\right)}\right. \\
& +\frac{1}{\left(m_{X}^{2}-p^{\prime 2}\right)\left(m_{J / \psi}^{2}-p^{2}\right)} \int_{s_{\phi}^{0}}^{\infty} d t \frac{\rho_{X \phi^{\prime}}\left(p^{\prime 2}, p^{2}, t\right)}{t-q^{2}} \\
& +\frac{1}{\left(m_{X}^{2}-p^{\prime 2}\right)\left(m_{\phi}^{2}-q^{2}\right)} \int_{s_{J / \psi}^{0}}^{\infty} d t \frac{\rho_{X \psi^{\prime}}\left(p^{\prime 2}, t, q^{2}\right)}{t-p^{2}} \\
& +\frac{1}{\left(m_{J / \psi}^{2}-p^{2}\right)\left(m_{\phi}^{2}-q^{2}\right)} \\
& \left.\times \int_{s_{X}^{0}}^{\infty} d t \frac{\rho_{X^{\prime} J / \psi}\left(t, p^{2}, q^{2}\right)+\rho_{X^{\prime} \phi}\left(t, p^{2}, q^{2}\right)}{t-p^{\prime 2}}+\cdots\right\} \\
& \left(\varepsilon_{\alpha \beta \mu \lambda} p^{\lambda}+\cdots\right)+\cdots, \\
& =\Pi\left(p^{\prime 2}, p^{2}, q^{2}\right) \varepsilon_{\alpha \beta \mu \lambda} p^{\lambda}+\cdots
\end{aligned}
$$

where $p^{\prime}=p+q$, the $g_{X J / \psi \phi}$ is the hadronic coupling constant defined by

$$
\left\langle J / \psi(p, \xi) \phi(q, \zeta) \mid X\left(p^{\prime}, \varepsilon\right)\right\rangle=i g_{X J / \psi \phi} \varepsilon^{\lambda \tau \rho \theta} p_{\lambda}^{\prime} \varepsilon_{\tau} \xi_{\rho}^{*} \zeta_{\theta}^{*},
$$

the four functions $\rho_{X \phi^{\prime}}\left(p^{\prime 2}, p^{2}, t\right), \rho_{X \psi^{\prime}}\left(p^{\prime 2}, t, q^{2}\right), \rho_{X^{\prime} J / \psi}$ $\left(t^{\prime}, p^{2}, q^{2}\right)$ and $\rho_{X^{\prime} \phi}\left(t^{\prime}, p^{2}, q^{2}\right)$ have complex dependence 

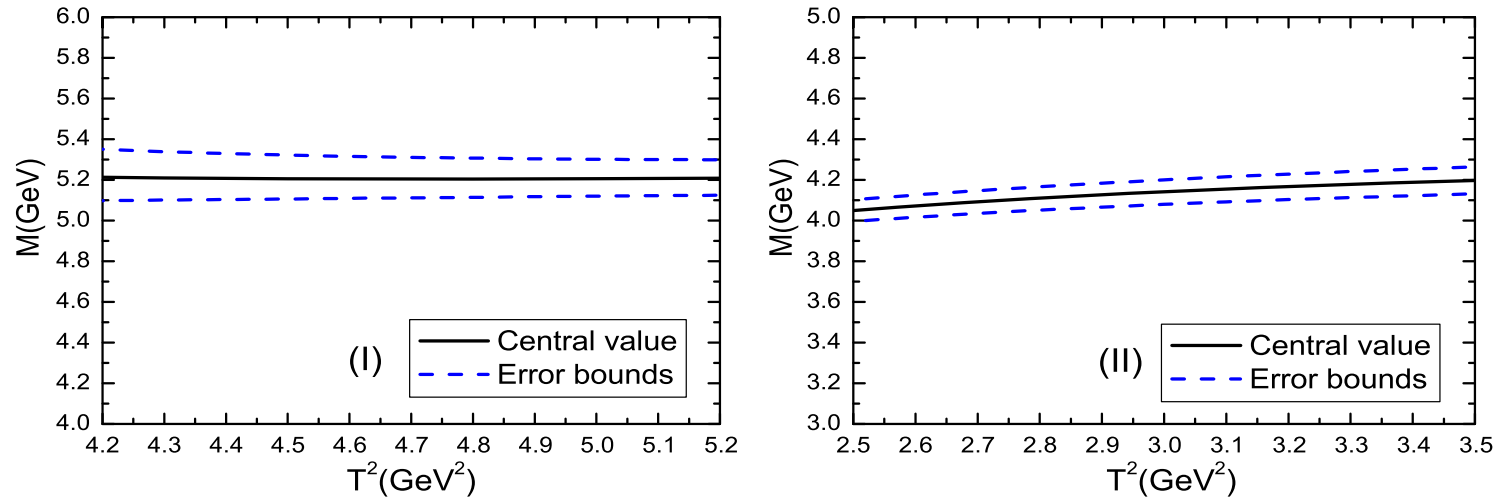

Fig. 1 The masses of the axialvector tetraquark states with variations of the Borel parameters $T^{2}$, where the (I) and (II) correspond to the currents $J_{\mu}^{1}(x)$ and $J_{\mu}^{2}(x)$, respectively
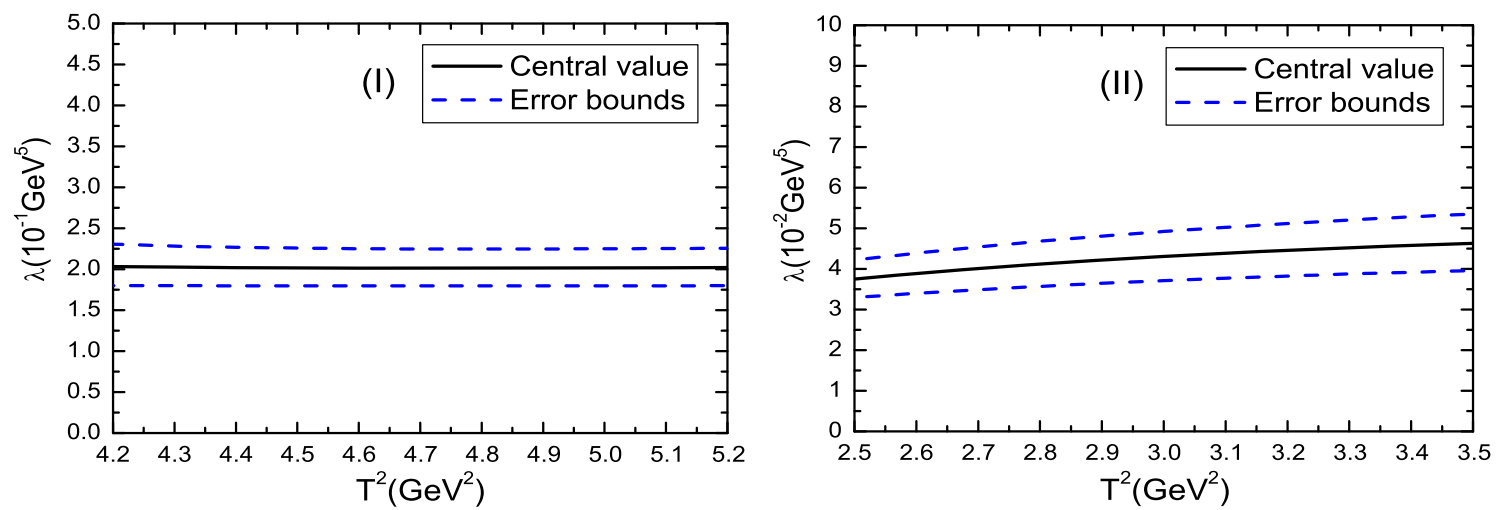

Fig. 2 The pole residues of the axialvector tetraquark states with variations of the Borel parameters $T^{2}$, where the (I) and (II) correspond to the currents $J_{\mu}^{1}(x)$ and $J_{\mu}^{2}(x)$, respectively

on the transitions between the ground states and the higher resonances or the continuum states.

In this article, we choose the tensor structure $\varepsilon_{\alpha \beta \mu \lambda} p^{\lambda}$ to study the $g_{X J / \psi \phi}$ to avoid the contaminations from the relevant scalar and pseudoscalar mesons according to the non-vanishing coupling constants,

$$
\begin{aligned}
\left\langle 0\left|J_{\alpha}^{J / \psi}(0)\right| \chi_{c 0}(p)\right\rangle & =f_{\chi_{c 0}} p_{\alpha}, \\
\left\langle 0\left|J_{\beta}^{\phi}(0)\right| f_{0}(q)\right\rangle & =f_{f_{0}} q \beta, \\
\left\langle X_{0}\left(p^{\prime}\right)\left|J_{\mu}^{\dagger}(0)\right| 0\right\rangle & =-i \lambda_{X_{0}} p_{\mu}^{\prime},
\end{aligned}
$$

where the $f_{\chi_{c 0}}, f_{f_{0}}$ and $\lambda_{X_{0}}$ are the decay constants of the $\chi_{c 0}(3414), f_{0}(980)$ and $X_{0}\left(J^{P}=0^{-}\right)$, respectively.

We introduce the parameters $C_{X \phi^{\prime}}, C_{X \psi^{\prime}}, C_{X^{\prime} \phi}$ and $C_{X^{\prime} J / \psi}$ to parameterize the net effects,

$$
\begin{aligned}
C_{X \phi^{\prime}} & =\int_{s_{\phi}^{0}}^{\infty} d t \frac{\rho_{X \phi^{\prime}}\left(p^{2}, p^{2}, t\right)}{t-q^{2}}, \\
C_{X \psi^{\prime}} & =\int_{s_{J / \psi}^{0}}^{\infty} d t \frac{\rho_{X \psi^{\prime}}\left(p^{\prime 2}, t, q^{2}\right)}{t-p^{2}}, \\
C_{X^{\prime} \phi} & =\int_{s_{X}^{0}}^{\infty} d t \frac{\rho_{X^{\prime} \phi}\left(t, p^{2}, q^{2}\right)}{t-p^{\prime 2}},
\end{aligned}
$$

$C_{X^{\prime} J / \psi}=\int_{s_{X}^{0}}^{\infty} d t \frac{\rho_{X^{\prime} J / \psi}\left(t, p^{2}, q^{2}\right)}{t-p^{\prime 2}}$.

Then the correlation function $\Pi\left(p^{\prime 2}, p^{2}, q^{2}\right)$ on the phenomenological side can be written as

$$
\begin{aligned}
& \Pi\left(p^{\prime 2}, p^{2}, q^{2}\right) \\
& =\frac{f_{\phi} m_{\phi} f_{J / \psi} m_{J / \psi} \lambda_{X} g_{X J / \psi \phi}}{\left(m_{X}^{2}-p^{\prime 2}\right)\left(m_{J / \psi}^{2}-p^{2}\right)\left(m_{\phi}^{2}-q^{2}\right)} \\
& \quad+\frac{C_{X \phi}}{\left(m_{X}^{2}-p^{\prime 2}\right)\left(m_{J / \psi}^{2}-p^{2}\right)} \\
& \quad+\frac{C_{X J / \psi}}{\left(m_{X}^{2}-p^{\prime 2}\right)\left(m_{\phi}^{2}-q^{2}\right)}+\frac{C_{X^{\prime} J / \psi}+C_{X^{\prime} \phi}}{\left(m_{J / \psi}^{2}-p^{2}\right)\left(m_{\phi}^{2}-q^{2}\right)} \\
& \quad+\cdots .
\end{aligned}
$$

Now we carry out the operator product expansion up to the vacuum condensates of dimension 5 and neglect the tiny contributions of the gluon condensate. The correlation function $\Pi_{Q C D}\left(p^{\prime 2}, p^{2}, q^{2}\right)$ can be written as 


$$
\begin{aligned}
& \Pi_{Q C D}\left(p^{\prime 2}, p^{2}, q^{2}\right) \\
& =\int_{4 m_{c}^{2}}^{s_{J / \psi}^{0}} d s \int_{0}^{u_{\phi}^{0}} d u \frac{\rho_{Q C D}\left(p^{\prime 2}, s, u\right)}{\left(s-p^{2}\right)\left(u-q^{2}\right)}+\cdots,
\end{aligned}
$$

through dispersion relation, where the $\rho_{Q C D}\left(p^{\prime 2}, s, u\right)$ is the QCD spectral density,

$$
\begin{aligned}
& \rho_{Q C D}\left(p^{\prime 2}, s, u\right) \\
& =\lim _{\epsilon_{2} \rightarrow 0} \lim _{\epsilon_{1} \rightarrow 0} \frac{\operatorname{Im}_{s} \operatorname{Im}_{u} \Pi_{Q C D}\left(p^{\prime 2}, s+i \epsilon_{2}, u+i \epsilon_{1}\right)}{\pi^{2}},
\end{aligned}
$$

we introduce the subscript $Q C D$ to denote the $Q C D$ side.

We rewrite the correlation function $\Pi_{H}\left(p^{\prime 2}, p^{2}, q^{2}\right)$ on the hadron side as

$$
\begin{aligned}
& \Pi_{H}\left(p^{\prime 2}, p^{2}, q^{2}\right) \\
& =\int_{\left(m_{J / \psi}+m_{\phi}\right)^{2}}^{s_{X}^{0}} d s^{\prime} \int_{4 m_{c}^{2}}^{s_{J / \psi}^{0}} d s \int_{0}^{u_{\phi}^{0}} d u \frac{\rho_{H}\left(s^{\prime}, s, u\right)}{\left(s^{\prime}-p^{\prime 2}\right)\left(s-p^{2}\right)\left(u-q^{2}\right)} \\
& \quad+\cdots,
\end{aligned}
$$

through dispersion relation, where the $\rho_{H}\left(s^{\prime}, s, u\right)$ is the hadronic spectral density,

$$
\begin{aligned}
& \rho_{H}\left(s^{\prime}, s, u\right)=\lim _{\epsilon_{3} \rightarrow 0} \lim _{\epsilon_{2} \rightarrow 0} \lim _{\epsilon_{1} \rightarrow 0} \\
& \times \frac{\operatorname{Im}_{s^{\prime}} \operatorname{Im}_{s} \operatorname{Im}_{u} \Pi_{H}\left(s^{\prime}+i \epsilon_{3}, s+i \epsilon_{2}, u+i \epsilon_{1}\right)}{\pi^{3}},
\end{aligned}
$$

we introduce the subscript $H$ to denote the hadron side. However, on the QCD side, the QCD spectral density $\rho_{Q C D}\left(s^{\prime}, s, u\right)$ does not exist,

$$
\begin{aligned}
& \rho_{Q C D}\left(s^{\prime}, s, u\right)=\lim _{\epsilon_{3} \rightarrow 0} \lim _{\epsilon_{2} \rightarrow 0} \lim _{\epsilon_{1} \rightarrow 0} \\
& \times \frac{\operatorname{Im}_{s^{\prime}} \operatorname{Im}_{s} \operatorname{Im}_{u} \Pi_{Q C D}\left(s^{\prime}+i \epsilon_{3}, s+i \epsilon_{2}, u+i \epsilon_{1}\right)}{\pi^{3}}=0,
\end{aligned}
$$

because

$\lim _{\epsilon_{3} \rightarrow 0} \frac{\operatorname{Im}_{s^{\prime}} \Pi_{Q C D}\left(s^{\prime}+i \epsilon_{3}, p^{2}, q^{2}\right)}{\pi}=0$.

We math the hadron side of the correlation function with the QCD side of the correlation function, and carry out the integral over $d s^{\prime}$ firstly to obtain the solid duality [42],

$$
\begin{aligned}
& \int_{\Delta_{s}^{2}}^{s^{0}} d s \int_{\Delta_{u}^{2}}^{u^{0}} d u \frac{\rho_{Q C D}\left(p^{\prime 2}, s, u\right)}{\left(s-p^{2}\right)\left(u-q^{2}\right)} \\
& \quad=\int_{\Delta_{s}^{2}}^{s^{0}} d s \int_{\Delta_{u}^{2}}^{u^{0}} d u \frac{1}{\left(s-p^{2}\right)\left(u-q^{2}\right)}\left[\int_{\Delta^{2}}^{\infty} d s^{\prime} \frac{\rho_{H}\left(s^{\prime}, s, u\right)}{s^{\prime}-p^{\prime 2}}\right],
\end{aligned}
$$

the $\Delta_{s}^{2}$ and $\Delta_{u}^{2}$ denote the thresholds $4 m_{c}^{2}$ and 0 , the $\Delta^{2}$ denotes the threshold $\left(m_{J / \psi}+m_{\phi}\right)^{2}$. Now we write the quarkhadron duality explicitly,

$$
\begin{aligned}
& \int_{4 m_{c}^{2}}^{s_{J / \psi}^{0}} d s \int_{0}^{u_{\phi}^{0}} d u \frac{\rho_{Q C D}\left(p^{\prime 2}, s, u\right)}{\left(s-p^{2}\right)\left(u-q^{2}\right)} \\
& =\int_{4 m_{c}^{2}}^{s_{J / \psi}^{0}} d s \int_{0}^{u_{\phi}^{0}} d u \int_{\left(m_{J / \psi}+m_{\phi}\right)^{2}}^{\infty} d s^{\prime} \frac{\rho_{H}\left(s^{\prime}, s, u\right)}{\left(s^{\prime}-p^{\prime 2}\right)\left(s-p^{2}\right)\left(u-q^{2}\right)} \\
& =\frac{f_{\phi} m_{\phi} f_{J / \psi} m_{J / \psi} \lambda_{X} g_{X J / \psi \phi}}{\left(m_{X}^{2}-p^{\prime 2}\right)\left(m_{J / \psi}^{2}-p^{2}\right)\left(m_{\phi}^{2}-q^{2}\right)} \\
& \quad+\frac{C_{X^{\prime} J / \psi}+C_{X^{\prime} \phi}}{\left(m_{J / \psi}^{2}-p^{2}\right)\left(m_{\phi}^{2}-q^{2}\right)} .
\end{aligned}
$$

No approximation is needed, we do not need the continuum threshold parameter $s_{X}^{0}$ in the $s^{\prime}$ channel. The present approach was introduced in Ref. [42].

In numerical calculations, we take the functions $C_{X \phi^{\prime}}$, $C_{X \psi^{\prime}}, C_{X^{\prime} \phi}$ and $C_{X^{\prime} J / \psi}$ as free parameters, and choose the suitable values to eliminate the contaminations from the higher resonances and continuum states to obtain the stable QCD sum rules with the variations of the Borel parameters. We set $p^{\prime 2}=p^{2}$ and perform the double Borel transform with respect to the variables $P^{2}=-p^{2}$ and $Q^{2}=-q^{2}$, respectively to obtain the QCD sum rules,

$$
\begin{aligned}
& \frac{f_{\phi} m_{\phi} f_{J / \psi} m_{J / \psi} \lambda_{X} g_{X J / \psi \phi}}{m_{X}^{2}-m_{J / \psi}^{2}}\left[\exp \left(-\frac{m_{J / \psi}^{2}}{T_{1}^{2}}\right)\right. \\
& \left.-\exp \left(-\frac{m_{X}^{2}}{T_{1}^{2}}\right)\right] \exp \left(-\frac{m_{\phi}^{2}}{T_{2}^{2}}\right) \\
& +\left(C_{X^{\prime} J / \psi}+C_{X^{\prime} \phi}\right) \exp \left(-\frac{m_{J / \psi}^{2}}{T_{1}^{2}}-\frac{m_{\phi}^{2}}{T_{2}^{2}}\right) \\
& =-\frac{1}{16 \sqrt{2} \pi^{4}} \int_{4 m_{c}^{2}}^{s_{s / \psi}^{0}} d s \int_{0}^{s_{\phi}^{0}} d u u \sqrt{1-\frac{4 m_{c}^{2}}{s}}\left(m_{c}-\frac{m_{s}}{2}\right. \\
& \left.-\frac{m_{s} m_{c}^{2}}{s}\right) \exp \left(-\frac{s}{T_{1}^{2}}-\frac{u}{T_{2}^{2}}\right) \\
& +\frac{m_{s} m_{c}\langle\bar{s} s\rangle}{2 \sqrt{2} \pi^{2}} \int_{4 m_{c}^{2}}^{s_{J / \psi}^{0}} d s \sqrt{1-\frac{4 m_{c}^{2}}{s}} \exp \left(-\frac{s}{T_{1}^{2}}\right) \\
& -\frac{\left\langle\bar{s} g_{s} \sigma G s\right\rangle}{36 \sqrt{2} \pi^{2}} \int_{4 m_{c}^{2}}^{s_{J / \psi}^{0}} d s \sqrt{1-\frac{4 m_{c}^{2}}{s}} \frac{s+2 m_{c}^{2}}{s} \exp \left(-\frac{s}{T_{1}^{2}}\right) \\
& \quad-\frac{m_{s} m_{c}\left\langle\bar{s} g_{s} \sigma G s\right\rangle}{24 \sqrt{2} \pi^{2} T_{2}^{2}} \int_{4 m_{c}^{2}}^{s_{J / \psi}^{0}} d s \sqrt{1-\frac{4 m_{c}^{2}}{s}} \exp \left(-\frac{s}{T_{1}^{2}}\right) \\
& \quad-\frac{m_{s} m_{c}\left\langle\bar{s} g_{s} \sigma G s\right\rangle}{16 \sqrt{2} \pi^{2}} \int_{4 m_{c}^{2}}^{s_{J / \psi}^{0}} d s \frac{1}{\sqrt{s^{2}-4 s m_{c}^{2}}} \exp \left(-\frac{s}{T_{1}^{2}}\right) .
\end{aligned}
$$

In calculations, we observe that there appears divergence due to the endpoint $s=4 m_{c}^{2}$, we can avoid the endpoint divergence with the simple replacement $\frac{1}{\sqrt{s^{2}-4 s m_{c}^{2}}} \rightarrow$ $\frac{1}{\sqrt{s^{2}-4 s m_{c}^{2}+4 m_{s}^{2} \mathrm{GeV}^{2}}}$ by adding a small squared $s$-quark mass $4 m_{s}^{2}$.

The hadronic parameters are taken as $m_{\phi}=1.019461 \mathrm{GeV}$, $m_{J / \psi}=3.0969 \mathrm{GeV}$ [8], $f_{J / \psi}=0.418 \mathrm{GeV}$ [43], $f_{\phi}=$ $0.253 \mathrm{GeV}, \sqrt{s_{\phi}^{0}}=1.5 \mathrm{GeV}[44], \sqrt{s_{J / \psi}^{0}}=3.6 \mathrm{GeV}$, 


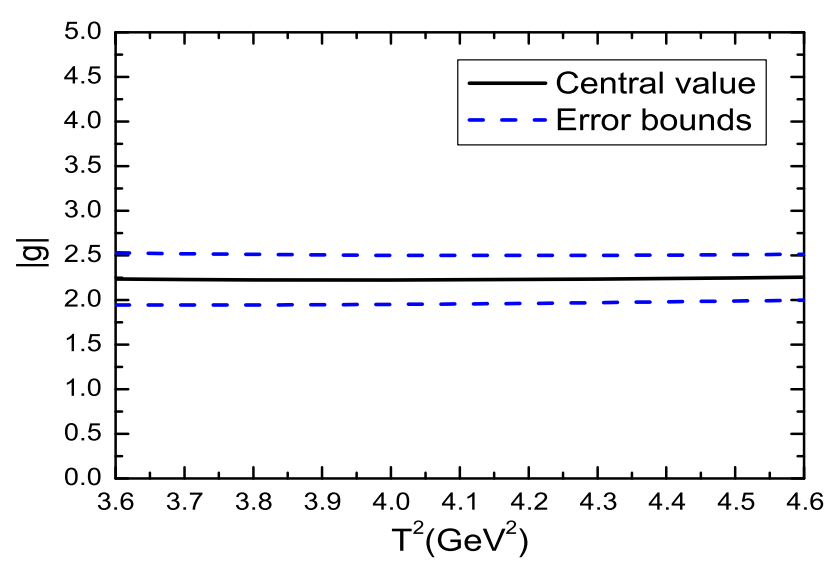

Fig. 3 The hadronic coupling constant $g_{X J / \psi \phi}$ with variation of the Borel parameter $T^{2}$

$M_{X}=4146.5 \mathrm{MeV}$ [6,7], $\lambda_{X}=4.30 \times 10^{-2} \mathrm{GeV}^{5}$. At the QCD side, we take the energy scale of the QCD spectral density to be $\mu=2 \mathrm{GeV}$, just like in the two-point QCD sum rules. Then we set the Borel parameters to be $T_{1}^{2}=T_{2}^{2}=T^{2}$ for simplicity. The unknown parameters are chosen as $C_{X^{\prime} J / \psi}+C_{X^{\prime} \phi}=-0.00261 \mathrm{GeV}^{7}$ to obtain platform in the Borel window $T^{2}=(3.6-4.6) \mathrm{GeV}^{2}$.

In Fig. 3, we plot the hadronic coupling constant $g_{X J / \psi \phi}$ with variation of the Borel parameter $T^{2}$. From the figure, we can see that there appears platform in the Borel window indeed. After taking into account the uncertainties of the input parameters, we obtain the hadronic coupling constant $g_{X J / \psi \phi}$,

$g_{X J / \psi \phi}=-(2.23 \pm 0.29)$.

Now it is easy to obtain the decay width,

$$
\begin{aligned}
& \Gamma(X(4140) \rightarrow J / \psi \phi) \\
& =\frac{p\left(m_{X}, m_{J / \psi}, m_{\phi}\right)}{24 \pi m_{X}^{2}} g_{X J / \psi \phi}^{2}\left\{\frac{\left(m_{X}^{2}-m_{\phi}^{2}\right)^{2}}{2 m_{J / \psi}^{2}}\right. \\
& \left.\quad+\frac{\left(m_{X}^{2}-m_{J / \psi}^{2}\right)^{2}}{2 m_{\phi}^{2}}+4 m_{X}^{2}-\frac{m_{J / \psi}^{2}+m_{\phi}^{2}}{2}\right\} \\
& =86.9 \pm 22.6 \mathrm{MeV},
\end{aligned}
$$

where $p(a, b, c)=\frac{\sqrt{\left[a^{2}-(b+c)^{2}\right]\left[a^{2}-(b-c)^{2}\right]}}{2 a}$. The width $\Gamma(X(4140) \rightarrow J / \psi \phi)=86.9 \pm 22.6 \mathrm{MeV}$ is in excellent agreement with the experimental data $83 \pm 21_{-14}^{+21} \mathrm{MeV}$ from the LHCb collaboration [6,7]. The present work supports assigning the $X(4140)$ to be the $[s c]_{T}[\bar{s} \bar{c}]_{V}-[s c]_{V}[\bar{s} \bar{c}]_{T}$ tetraquark state with $J^{P C}=1^{++}$.

\section{Conclusion}

In this article, we construct both the $[s c]_{T}[\bar{s} \bar{c}]_{A}+[s c]_{A}[\bar{s} \bar{c}]_{T}$ type and $[s c]_{T}[\bar{s} \bar{c}]_{V}-[s c]_{V}[\bar{s} \bar{c}]_{T}$ type axialvector currents with $J^{P C}=1^{++}$to study the mass of the $X(4140)$ with the QCD sum rules by carrying out the operator product expansion up to the vacuum condensates of dimension 10 and take the energy scale formula $\mu=\sqrt{M_{X / Y / Z}^{2}-\left(2 \mathbb{M}_{C}\right)^{2}}$ to determine the ideal energy scales of the QCD spectral densities. The predicted masses support assigning the $X(4140)$ to be the $[s c]_{T}[\bar{s} \bar{c}]_{V}-[s c]_{V}[\bar{s} \bar{c}]_{T}$ type axialvector tetraquark state. Then we calculate the hadronic coupling constant $g_{X J / \psi \phi}$ with the QCD sum rules based on the solid quark-hadron duality, and obtain the decay width $\Gamma(X(4140) \rightarrow J / \psi \phi)=$ $86.9 \pm 22.6 \mathrm{MeV}$, which is in excellent agreement with the experimental data $83 \pm 21_{-14}^{+21} \mathrm{MeV}$ from the LHCb collaboration. In summary, the present work supports assigning the $X(4140)$ to be the $[s c]_{T}[\bar{s} \bar{c}]_{V}-[s c]_{V}[\bar{s} \bar{c}]_{T}$ type tetraquark state with $J^{P C}=1^{++}$.

Acknowledgements This work is supported by National Natural Science Foundation, Grant Number 11775079.

Data Availability Statement This manuscript has no associated data or the data will not be deposited. [Authors' comment: All data included in this manuscript are available upon request by contacting with the corresponding author.]

Open Access This article is distributed under the terms of the Creative Commons Attribution 4.0 International License (http://creativecomm ons.org/licenses/by/4.0/), which permits unrestricted use, distribution, and reproduction in any medium, provided you give appropriate credit to the original author(s) and the source, provide a link to the Creative Commons license, and indicate if changes were made. Funded by SCOAP . $^{3}$

\section{Appendix}

The explicit expressions of the QCD spectral densities $\rho_{0}(s)$, $\rho_{3}(s), \rho_{5}(s), \rho_{6}(s), \rho_{7}(s), \rho_{8}(s)$ and $\rho_{10}(s)$,

$$
\begin{gathered}
\rho_{0}(s) \\
=\frac{3}{1024 \pi^{6}} \int d y d z y z(1-y-z)^{2}\left(s-\bar{m}_{c}^{2}\right)^{3}\left(5 s-\bar{m}_{c}^{2}\right) \\
\quad-\frac{m_{c}^{2}}{768 \pi^{6}} \int d y d z(5+y+z)(1-y-z)^{2}\left(s-\bar{m}_{c}^{2}\right)^{3} \\
\quad-\frac{3 m_{s} m_{c}}{256 \pi^{6}} \int d y d z y(1-y-z)^{2}\left(s-\bar{m}_{c}^{2}\right)^{2}\left(3 s-\bar{m}_{c}^{2}\right), \\
\rho_{3}(s)=\frac{m_{c}\langle\bar{s} s\rangle}{32 \pi^{4}} \int d y d z y(1-y-z)\left(s-\bar{m}_{c}^{2}\right)\left(7 s-3 \bar{m}_{c}^{2}\right) \\
\quad+\frac{m_{s}\langle\bar{s} s\rangle}{32 \pi^{4}} \int d y d z y z\left(s-\bar{m}_{c}^{2}\right)\left(23 s-9 \bar{m}_{c}^{2}\right) \\
\quad-\frac{m_{s} m_{c}^{2}\langle\bar{s} s\rangle}{16 \pi^{4}} \int d y d z(7+y+z)\left(s-\bar{m}_{c}^{2}\right), \\
\rho_{4}(s)=\frac{m_{c}^{2}}{1152 \pi^{4}}\left\langle\frac{\alpha_{s} G G}{\pi}\right\rangle
\end{gathered}
$$




$$
\begin{aligned}
& \times \int d y d z \frac{\left[y^{2}+(z-1) y-9 z\right](1-y-z)^{2}}{y^{2}}\left(2 s-\bar{m}_{c}^{2}\right) \\
& +\frac{m_{c}^{2}}{96 \pi^{4}}\left\langle\frac{\alpha_{s} G G}{\pi}\right\rangle \int d y d z \frac{(1-y-z)^{2}}{y^{2}}\left[\frac{y s}{2}-(1-y)\left(s-\bar{m}_{c}^{2}\right)\right] \\
& +\frac{m_{c}^{2}}{576 \pi^{4}}\left\langle\frac{\alpha_{s} G G}{\pi}\right\rangle \int d y d z \frac{(1-y-z)^{3}(1-y)}{y^{2}}\left(s-\bar{m}_{c}^{2}\right) \\
& +\frac{m_{s} m_{c}^{3}}{256 \pi^{4}}\left\langle\frac{\alpha_{s} G G}{\pi}\right\rangle \int d y d z \frac{(1-y-z)^{2}}{y^{2}}\left[1+\frac{2 s}{3} \delta\left(s-\bar{m}_{c}^{2}\right)\right] \\
& +\frac{m_{s} m_{c}}{128 \pi^{4}}\left\langle\frac{\alpha_{s} G G}{\pi}\right\rangle \int d y d z \frac{z(1-y-z)^{2}}{y^{2}}\left[\left(\frac{3 y}{2}-1\right)\left(s-\bar{m}_{c}^{2}\right)\right. \\
& \left.-\frac{2 s}{3}+\frac{11 y s}{6}+\frac{y s^{2}}{3} \delta\left(s-\bar{m}_{c}^{2}\right)\right] \\
& +\frac{1}{1536 \pi^{4}}\left\langle\frac{\alpha_{s} G G}{\pi}\right\rangle \int d y d z\left[(1-y-z)^{2}+2 y z\right] s\left(s-\bar{m}_{c}^{2}\right) \\
& +\frac{m_{c}^{2}}{3072 \pi^{4}}\left\langle\frac{\alpha_{s} G G}{\pi}\right\rangle \int d y d z \frac{(6 y+3 z+9)(1-y-z)}{y}\left(s-\bar{m}_{c}^{2}\right) \\
& +\frac{m_{s} m_{c}}{512 \pi^{4}}\left\langle\frac{\alpha_{s} G G}{\pi}\right\rangle \int d y d z \frac{(2 z-3 y)(1-y-z)}{y}\left(5 s-3 \bar{m}_{c}^{2}\right) \\
& +\frac{m_{s} m_{c}}{3072 \pi^{4}}\left\langle\frac{\alpha_{s} G G}{\pi}\right\rangle \int d y d z \frac{(1-y-z)^{2}}{z}\left(5 s-3 \bar{m}_{c}^{2}\right) \\
& -\frac{m_{c}^{2}}{512 \pi^{4}}\left\langle\frac{\alpha_{s} G G}{\pi}\right\rangle \int d y d z\left(s-\bar{m}_{c}^{2}\right) \\
& -\frac{7 m_{c}^{2}}{3072 \pi^{4}}\left\langle\frac{\alpha_{s} G G}{\pi}\right\rangle \int d y d z \frac{(1-y-z)^{2}}{y z}\left(s-\bar{m}_{c}^{2}\right) \\
& +\frac{13 m_{c}^{2}}{18432 \pi^{4}}\left\langle\frac{\alpha_{s} G G}{\pi}\right\rangle \int d y d z \frac{(1-y-z)^{3}}{y z}\left(s-\bar{m}_{c}^{2}\right) \text {, }
\end{aligned}
$$

$$
\begin{aligned}
& -\frac{m_{s} m_{c}^{2}\langle\bar{s} s\rangle}{144 \pi^{2} T^{2}}\left\langle\frac{\alpha_{s} G G}{\pi}\right\rangle \int d y d z \frac{(1-y-z)}{y} s \delta\left(s-\bar{m}_{c}^{2}\right) \\
& +\frac{m_{c}\langle\bar{s} s\rangle}{128 \pi^{2}}\left\langle\frac{\alpha_{s} G G}{\pi}\right\rangle \int d y d z \frac{3 y-2 z}{y}\left[1+\frac{2 s}{3} \delta\left(s-\bar{m}_{c}^{2}\right)\right] \\
& +\frac{m_{s}\langle\bar{s} s\rangle}{768 \pi^{2}}\left\langle\frac{\alpha_{s} G G}{\pi}\right\rangle \int d y s \delta\left(s-\tilde{m}_{c}^{2}\right) \\
& -\frac{m_{s} m_{c}^{2}\langle\bar{s} s\rangle}{768 \pi^{2}}\left\langle\frac{\alpha_{s} G G}{\pi}\right\rangle \int d y d z \frac{1}{y} \delta\left(s-\bar{m}_{c}^{2}\right) \\
& -\frac{m_{c}\langle\bar{s} s\rangle}{384 \pi^{2}}\left\langle\frac{\alpha_{s} G G}{\pi}\right\rangle \int d y d z \frac{(1-y-z)}{z}\left[1+\frac{2 s}{3} \delta\left(s-\bar{m}_{c}^{2}\right)\right] \\
& +\frac{m_{s}\langle\bar{s} s\rangle}{128 \pi^{2}}\left\langle\frac{\alpha_{s} G G}{\pi}\right\rangle \int d y d z\left[1+\frac{8 s}{9} \delta\left(s-\bar{m}_{c}^{2}\right)\right] \\
& -\frac{m_{s} m_{c}^{2}\langle\bar{s} s\rangle}{2304 \pi^{2}}\left\langle\frac{\alpha_{s} G G}{\pi}\right\rangle \int d y d z \frac{25+13 y+13 z}{y z} \delta\left(s-\bar{m}_{c}^{2}\right) \\
& +\frac{m_{c}\langle\bar{s} s\rangle}{192 \pi^{2}}\left\langle\frac{\alpha_{s} G G}{\pi}\right\rangle \int d y y[1 \\
& \left.+\frac{2 s}{3} \delta\left(s-\widetilde{m}_{c}^{2}\right)\right] \\
& +\frac{m_{s}\langle\bar{s} s\rangle}{32 \pi^{2}}\left\langle\frac{\alpha_{s} G G}{\pi}\right\rangle \int d y y(1-y)[1 \\
& \left.+\left(\frac{7 s}{9}+\frac{2 s^{2}}{9 T^{2}}\right) \delta\left(s-\tilde{m}_{c}^{2}\right)\right] \\
& -\frac{m_{s} m_{c}^{2}\langle\bar{s} s\rangle}{96 \pi^{2}}\left\langle\frac{\alpha_{s} G G}{\pi}\right\rangle \int d y\left(1+\frac{s}{T^{2}}\right) \delta\left(s-\tilde{m}_{c}^{2}\right) \\
&
\end{aligned}
$$

$\rho_{5}(s)$

$$
\begin{aligned}
= & -\frac{m_{c}\left\langle\bar{s} g_{s} \sigma G s\right\rangle}{64 \pi^{4}} \int d y d z y\left(5 s-3 \bar{m}_{c}^{2}\right) \\
& -\frac{m_{s}\left\langle\bar{s} g_{s} \sigma G s\right\rangle}{32 \pi^{4}} \int d y y(1-y)\left(7 s-4 \tilde{m}_{c}^{2}\right) \\
& +\frac{11 m_{s} m_{c}^{2}\left\langle\bar{s} g_{s} \sigma G s\right\rangle}{96 \pi^{4}} \int d y-\frac{m_{s} m_{c}^{2}\left\langle\bar{s} g_{s} \sigma G s\right\rangle}{96 \pi^{4}} \int d y d z \\
& +\frac{m_{c}\left\langle\bar{s} g_{s} \sigma G s\right\rangle}{768 \pi^{4}} \int d y d z \frac{5 y^{2}-3(z+2) y-9(z-1) z}{y} \\
& \times\left(5 s-3 \bar{m}_{c}^{2}\right)-\frac{m_{s}\left\langle\bar{s} g_{s} \sigma G s\right\rangle}{256 \pi^{4}} \int d y d z z\left(5 s-3 \bar{m}_{c}^{2}\right), \\
\rho_{6}(s) & =-\frac{\langle\bar{s} s\rangle^{2}}{12 \pi^{2}} \int d y y(1-y)\left(5 s-3 \tilde{m}_{c}^{2}\right)+\frac{m_{c}^{2}\langle\bar{s} s\rangle^{2}}{4 \pi^{2}} \int d y \\
& +\frac{m_{s} m_{c}\langle\bar{s} s\rangle^{2}}{8 \pi^{2}} \int d y y\left[1+\frac{2 s}{3} \delta\left(s-\widetilde{m}_{c}^{2}\right)\right],
\end{aligned}
$$

$\rho_{8}(s)$

$$
\begin{aligned}
= & \frac{\langle\bar{s} s\rangle\left\langle\bar{s} g_{s} \sigma G s\right\rangle}{8 \pi^{2}} \int d y y(1-y)\left[3+\left(\frac{7 s}{3}\right.\right. \\
& \left.\left.+\frac{2 s^{2}}{3 T^{2}}\right) \delta\left(s-\tilde{m}_{c}^{2}\right)\right] \\
& -\frac{m_{c}^{2}\langle\bar{s} s\rangle\left\langle\bar{s} g_{s} \sigma G s\right\rangle}{8 \pi^{2}} \int d y\left(1+\frac{s}{T^{2}}\right) \delta\left(s-\widetilde{m}_{c}^{2}\right) \\
& -\frac{5 m_{s} m_{c}\langle\bar{s} s\rangle\left\langle\bar{s} g_{s} \sigma G s\right\rangle}{144 \pi^{2}} \int d y y\left(1+\frac{3 s}{2 T^{2}}\right. \\
& \left.+\frac{s^{2}}{T^{4}}\right) \delta\left(s-\tilde{m}_{c}^{2}\right) \\
& +\frac{\langle\bar{s}\rangle\left\langle\bar{s} g_{s} \sigma G s\right\rangle}{128 \pi^{2}} \int d y\left[1+\frac{2 s}{3} \delta\left(s-\widetilde{m}_{c}^{2}\right)\right] \\
& +\frac{m_{s} m_{c}\langle\bar{s} s\rangle\left\langle\bar{s} g_{s} \sigma G s\right\rangle}{128 \pi^{2}} \int d y \frac{1-y}{y}\left(1+\frac{2 s}{T^{2}}\right) \delta\left(s-\tilde{m}_{c}^{2}\right) \\
& -\frac{m_{s} m_{c}\langle\bar{s} s\rangle\left\langle\bar{s} g_{s} \sigma G s\right\rangle}{192 \pi^{2}} \int d y\left(1+\frac{2 s}{T^{2}}\right) \delta\left(s-\tilde{m}_{c}^{2}\right),
\end{aligned}
$$

$$
\begin{aligned}
= & -\frac{m_{c}^{3}\langle\bar{s} s\rangle}{288 \pi^{2}}\left\langle\frac{\alpha_{s} G G}{\pi}\right\rangle \int d y d z \frac{(1-y-z)}{y^{2}}\left(1+\frac{2 s}{T^{2}}\right) \delta\left(s-\bar{m}_{c}^{2}\right) \\
& +\frac{m_{c}\langle\bar{s} s\rangle}{16 \pi^{2}}\left\langle\frac{\alpha_{s} G G}{\pi}\right\rangle \int d y d z \frac{z(1-y-z)}{y^{2}}\left[\frac{1}{3}\right. \\
& \left.-\frac{y}{2}+\left(\frac{2 s}{9}-\frac{7 y s}{18}-\frac{y s^{2}}{9 T^{2}}\right) \delta\left(s-\bar{m}_{c}^{2}\right)\right] \\
& -\frac{m_{s} m_{c}^{2}\langle\bar{s} s\rangle}{72 \pi^{2}}\left\langle\frac{\alpha_{s} G G}{\pi}\right\rangle \int d y d z \frac{z}{y^{2}}\left(1+\frac{7 s}{2 T^{2}}\right) \delta\left(s-\bar{m}_{c}^{2}\right) \\
& -\frac{m_{s} m_{c}^{2}\langle\bar{s} s\rangle}{18 \pi^{2}}\left\langle\frac{\alpha_{s} G G}{\pi}\right\rangle \int d y d z \frac{1}{y^{2}}\left(2-y-\frac{y s}{T^{2}}\right) \delta\left(s-\bar{m}_{c}^{2}\right) \\
& +\frac{m_{s} m_{c}^{2}\langle\bar{s} s\rangle}{72 \pi^{2}}\left\langle\frac{\alpha_{s} G G}{\pi}\right\rangle \int d y d z \frac{(1-y-z)(1-y)}{y^{2}} \delta\left(s-\bar{m}_{c}^{2}\right)
\end{aligned}
$$

$\rho_{10}(s)$

$$
\begin{aligned}
= & -\frac{\left\langle\bar{s} g_{s} \sigma G s\right\rangle^{2}}{32 \pi^{2}} \int d y y(1-y)\left(1+\frac{4 s}{3 T^{2}}\right. \\
& \left.+\frac{5 s^{2}}{6 T^{4}}-\frac{s^{3}}{6 T^{6}}\right) \delta\left(s-\widetilde{m}_{c}^{2}\right) \\
& -\frac{m_{s} m_{c}\left\langle\bar{s} g_{s} \sigma G s\right\rangle^{2}}{288 \pi^{2} T^{2}} \int d y y\left(1+\frac{s}{T^{2}}\right. \\
& \left.+\frac{s^{2}}{2 T^{4}}-\frac{s^{3}}{T^{6}}\right) \delta\left(s-\widetilde{m}_{c}^{2}\right) \\
& -\frac{m_{c}^{2}\langle\bar{s} s\rangle^{2}}{108 T^{2}}\left\langle\frac{\alpha_{s} G G}{\pi}\right\rangle \int d y \frac{(1-y)}{y^{2}}\left(1-\frac{2 s}{T^{2}}\right) \delta\left(s-\widetilde{m}_{c}^{2}\right)
\end{aligned}
$$




$$
\begin{aligned}
& -\frac{m_{c}^{2}\langle\bar{s} s\rangle^{2}}{18 T^{2}}\left\langle\frac{\alpha_{s} G G}{\pi}\right\rangle \int d y \frac{1}{y^{2}}\left(1-\frac{y s}{2 T^{2}}\right) \delta\left(s-\widetilde{m}_{c}^{2}\right) \\
& +\frac{m_{s} m_{c}^{3}\langle\bar{s} s\rangle^{2}}{144 T^{4}}\left\langle\frac{\alpha_{s} G G}{\pi}\right\rangle \int d y \frac{1}{y^{2}}\left(1-\frac{2 s}{3 T^{2}}\right) \delta\left(s-\widetilde{m}_{c}^{2}\right) \\
& +\frac{m_{s} m_{c}\langle\bar{s} s\rangle^{2}}{216 T^{2}}\left\langle\frac{\alpha_{s} G G}{\pi}\right\rangle \int d y \frac{1-y}{y^{2}}\left(\frac{y}{2}-1+\frac{2 s}{T^{2}}\right. \\
& \left.+\frac{y s}{2 T^{2}}-\frac{y s^{2}}{T^{4}}\right) \delta\left(s-\tilde{m}_{c}^{2}\right) \\
& +\frac{m_{s} m_{c}\langle\bar{s} s\rangle^{2}}{1728 T^{2}}\left\langle\frac{\alpha_{s} G G}{\pi}\right\rangle \int d y \frac{1}{1-y}\left(1-\frac{2 s}{T^{2}}\right) \delta\left(s-\widetilde{m}_{c}^{2}\right) \\
& -\frac{\langle\bar{s} s\rangle^{2}}{576}\left\langle\frac{\alpha_{s} G G}{\pi}\right\rangle \int d y\left(1-\frac{2 s}{T^{2}}\right) \delta\left(s-\widetilde{m}_{c}^{2}\right) \\
& -\frac{\left\langle\bar{s} g_{s} \sigma G s\right\rangle^{2}}{768 \pi^{2}} \int d y\left(1+\frac{3 s}{2 T^{2}}+\frac{s^{2}}{T^{4}}\right) \delta\left(s-\widetilde{m}_{c}^{2}\right) \\
& +\frac{m_{s} m_{c}\left\langle\bar{s} g_{s} \sigma G s\right\rangle^{2}}{768 \pi^{2} T^{2}} \int d y \frac{1-y}{y}\left(1+\frac{s}{T^{2}}\right. \\
& \left.-\frac{2 s^{2}}{T^{4}}\right) \delta\left(s-\tilde{m}_{c}^{2}\right) \\
& +\frac{m_{s} m_{c}\left\langle\bar{s} g_{s} \sigma G s\right\rangle^{2}}{1152 \pi^{2} T^{2}} \int d y\left(1+\frac{s}{T^{2}}\right. \\
& \left.-\frac{2 s^{2}}{T^{4}}\right) \delta\left(s-\tilde{m}_{c}^{2}\right) \\
& +\frac{7\left\langle\bar{s} g_{s} \sigma G s\right\rangle^{2}}{27648 \pi^{2}} \int d y\left(1+\frac{2 s}{T^{2}}\right) \delta\left(s-\tilde{m}_{c}^{2}\right) \\
& -\frac{\left.m_{s} m_{c}\right\rangle^{2}}{36}\left\langle\frac{\alpha_{s} G G}{\pi}\right\rangle \int d y y(1-y)\left(1+\frac{4 s}{3 T^{2}}\right. \\
& \hline s^{2} \\
& \hline T^{2}
\end{aligned}
$$

where $\int d y d z=\int_{y_{i}}^{y_{f}} d y \int_{z_{i}}^{1-y} d z, y_{f}=\frac{1+\sqrt{1-4 m_{c}^{2} / s}}{2}, y_{i}=$ $\frac{1-\sqrt{1-4 m_{c}^{2} / s}}{2}, z_{i}=\frac{y m_{c}^{2}}{y s-m_{c}^{2}}, \bar{m}_{c}^{2}=\frac{(y+z) m_{c}^{2}}{y z}, \tilde{m}_{c}^{2}=\frac{m_{c}^{2}}{y(1-y)}$, $\int_{y_{i}}^{y_{f}} d y \rightarrow \int_{0}^{1} d y, \int_{z_{i}}^{1-y} d z \rightarrow \int_{0}^{1-y} d z$, when the $\delta$ functions $\delta\left(s-\bar{m}_{c}^{2}\right)$ and $\delta\left(s-\tilde{m}_{c}^{2}\right)$ appear.
2. T. Aaltonen et al., Mod. Phys. Lett. A 32, 1750139 (2017)

3. S. Chatrchyan et al., Phys. Lett. B 734, 261 (2014)

4. V.M. Abazov et al., Phys. Rev. D 89, 012004 (2014)

5. V.M. Abazov et al., Phys. Rev. Lett. 115, 232001 (2015)

6. R. Aaij et al., Phys. Rev. Lett. 118, 022003 (2017)

7. R. Aaij et al., Phys. Rev. D 95, 012002 (2017)

8. M. Tanabashi et al., Phys. Rev. D 98, 030001 (2018)

9. F. Stancu, J. Phys. G 37, 075017 (2010)

10. Z.G. Wang, Y.F. Tian, Int. J. Mod. Phys. A 30, 1550004 (2015)

11. R.F. Lebed, A.D. Polosa, Phys. Rev. D 93, 094024 (2016)

12. L. Maiani, A.D. Polosa, V. Riquer, Phys. Rev. D 94, 054026 (2016)

13. R. Zhu, Phys. Rev. D 94, 054009 (2016)

14. Q. Fang Lu, Y.B. Dong, Phys. Rev. D 94, 074007 (2016)

15. J. Wu, Y.R. Liu, K. Chen, X. Liu, S.L. Zhu, Phys. Rev. D 94, 094031 (2016)

16. M.N. Anwar, J. Ferretti, E. Santopinto, Phys. Rev. D 98, 094015 (2018)

17. N. Mahajan, Phys. Lett. B 679, 228 (2009)

18. Z.G. Wang, Eur. Phys. J. C 63, 115 (2009)

19. Z.G. Wang, Z.C. Liu, X.H. Zhang, Eur. Phys. J. C 64, 373 (2009)

20. X.H. Liu, Phys. Lett. B 766, 117 (2017)

21. Z.G. Wang, Eur. Phys. J. C 77, 78 (2017)

22. Z.G. Wang, Eur. Phys. J. A 53, 19 (2017)

23. Z.G. Wang, Eur. Phys. J. C 76, 657 (2016)

24. W. Chen, S.L. Zhu, Phys. Rev. D 83, 034010 (2011)

25. H.X. Chen, E.L. Cui, W. Chen, X. Liu, S.L. Zhu, Eur. Phys. J. C 77, 160 (2017)

26. S.S. Agaev, K. Azizi, H. Sundu, Phys. Rev. 95, 114003 (2017)

27. W. Chen, H.X. Chen, X. Liu, T.G. Steele, S.L. Zhu, Phys. Rev. D 96, 114017 (2017)

28. Z.G. Wang, Eur. Phys. J. C 74, 2874 (2014)

29. Z.G. Wang, T. Huang, Nucl. Phys. A 930, 63 (2014)

30. Z.G. Wang, T. Huang, Eur. Phys. J. C 74, 2891 (2014)

31. Z.G. Wang, Eur. Phys. J. C 74, 2963 (2014)

32. Z.G. Wang, Eur. Phys. J. C 76, 70 (2016)

33. Z.G. Wang, T. Huang, Eur. Phys. J. C 76, 43 (2016)

34. Z.G. Wang, Nucl. Phys. B 913, 163 (2016)

35. Z.G. Wang, Eur. Phys. J. C 76, 387 (2016)

36. M.A. Shifman, A.I. Vainshtein, V.I. Zakharov, Nucl. Phys. B 147, 385 (1979)

37. M.A. Shifman, A.I. Vainshtein, V.I. Zakharov, Nucl. Phys. B 147, 448 (1979)

38. L.J. Reinders, H. Rubinstein, S. Yazaki, Phys. Rept. 127, 1 (1985)

39. Z.G. Wang, T. Huang, Phys. Rev. D 89, 054019 (2014)

40. P. Colangelo, A. Khodjamirian. arXiv:hep-ph/0010175

41. S. Narison, R. Tarrach, Phys. Lett. B 125, 217 (1983)

42. Z.G. Wang, J.X. Zhang, Eur. Phys. J. C 78, 14 (2018)

43. D. Becirevic, G. Duplancic, B. Klajn, B. Melic, F. Sanfilippo, Nucl. Phys. B 883, 306 (2014)

44. Z.G. Wang, Eur. Phys. J. C 77, 174 (2017)

\section{References}

1. T. Aaltonen et al., Phys. Rev. Lett. 102, 242002 (2009) 